\section{Tabagismo e consumo de álcool \\ em estudantes universitários: \\ prevalência e fatores associados}

\section{Smoking and alcohol consumption among university students: prevalence and associated factors}

Thiago Rozales Ramis'

Grégore Iven Mielke'

Esther Campos Habeyche'

Manoela Maciel Oliz'

Mario Renato Azevedo"

Pedro Curi Hallal"

'Acadêmicos da Escola Superior de Educação Física da Universidade Federal de Pelotas

"Professores Adjuntos da Escola Superior de Educação Física da Universidade Federal de Pelotas

Correspondência: Thiago Rozales Ramis. Escola Superior de Educação Física da Universidade Federal de Pelotas. Rua Luis de Camões, 625. Pelotas, RS- CEP 96055-630. E-mail:

Thiago.ramis@yahoo.com.br

\section{Resumo}

O presente estudo teve o objetivo de verificar a prevalência de tabagismo e consumo de álcool entre estudantes da Universidade Federal de Pelotas (UFPel), além de investigar os fatores associados a esses comportamentos. A amostra foi composta por 485 alunos que ingressaram na UFPel em 2008. Os alunos foram selecionados de maneira aleatória entre cursos de todas as áreas da UFPel e responderam a um questionário pré-testado na sala de aula, sob supervisão da equipe de pesquisa. Dos indivíduos entrevistados, 53,9\% eram do sexo feminino e $42,3 \%$ tinham menos de 20 anos. Em relação ao consumo de álcool, $75 \%$ da amostra o utilizam pelo menos uma vez por mês; a prevalência de risco para o alcoolismo foi de $6,2 \%$. Em relação ao tabagismo, $10,2 \%$ dos estudantes relataram fumar regularmente ou nos fins-de-semana. Além disso, mais de $90 \%$ dos fumantes e dos que consomem bebidas alcoólicas iniciaram o hábito antes de ingressar na universidade. O tabagismo apresentou uma relação direta com a idade e inversa com a autopercepção de saúde. Em relação ao álcool, estudantes que moram com amigos relataram um maior consumo. Os dados sugerem a necessidade de intervenções no meio acadêmico. Entretanto, cabe destacar que as ações para a população adolescente como um todo devam ser priorizadas, pois mais de $90 \%$ dos alunos adquiriram os hábitos antes de ingressarem na universidade, demonstrando que o início do consumo está ocorrendo em idades mais precoces.

Palavras-chaves: Consumo de álcool. Tabagismo. Universitários. Epidemiologia. Estudantes. Estudos transversais. 


\section{Abstract}

The study aimed to estimate the prevalence of smoking and alcohol intake among university students from the Federal University of Pelotas, Brazil (UFPel), as well as to investigate factors associated with both habits. The sample included 485 students who were admitted to the university in 2008. Students were sampled randomly across all schools of the UFPel campus, and answered a pre-tested questionnaire, which was administered in the classroom by a member of the research team. Of the individuals interviewed, $53.9 \%$ were females and $42.3 \%$ were under 20 years. Regarding alcohol intake, $75 \%$ used alcohol once a month or less frequently, and the prevalence of risk for abusive alcohol intake was $6.2 \%$. Regarding smoking, $10.2 \%$ reported smoking regularly or on weekends. More than $90 \%$ of those who smoked or used alcohol started before entering the university. Smoking was directly related to age and inversely related to self-rated health. In terms of alcohol intake, those who lived with friends were more likely to use it. Our data suggest the need for implementing strategies to promote healthy lifestyles among university students. However, the fact that more than $90 \%$ of individuals started to smoke or drink before entering the university suggests that interventions should target adolescents as a whole, and not only those who are university students, because onset of smoking and alcohol intake seems to occur at earlier ages.

Keywords: Alcohol Drinking. Smoking. Colleges. Epidemiology. Students. CrossSectional Studies.

\section{Introdução}

Nas últimas décadas, os padrões de morbimortalidade sofreram modificações profundas, sendo que a predominância das mortes deixou de ser por doenças infecto-contagiosas para ser decorrente de doenças ligadas ao estilo de vida. Esse processo é conhecido como transição epidemiológica e afetou inicialmente os países de renda alta, mas posteriormente chegou aos países de renda média e baixa, sendo que nesses o processo ocorreu de maneira acelerada ${ }^{1}$.

Entre os comportamentos prejudiciais à saúde, destaca-se o consumo de álcool, por ser um dos mais prevalentes na população, inclusive entre os jovens ${ }^{2}$. Os malefícios do consumo de álcool, especialmente em doses abusivas, são bem esclarecidos na literatura ${ }^{3}$.

Recentes levantamentos populacionais comprovam que o consumo de álcool é bastante relevante entre a população. Um estudo realizado pelo CEBRID (Centro Brasileiro de Informações sobre Drogas Psicotrópicas) em 107 cidades com mais de 200 mil habitantes mostrou que aproximadamente $68 \%$ da amostra consumiam álcool. Também foi evidenciado que 15,5\% das pessoas na faixa de 18 a 24 anos eram dependentes de álcool ${ }^{4}$.

Associado a isto, o tabagismo também é considerado um forte fator de risco para doenças e agravos não transmissíveis, sendo elevadas as taxas de morbimortalidade direta ou indiretamente ligadas ao consumo de cigarro ${ }^{5}$. O consumo de tabaco é a causa de aproximadamente 4,9 milhões de mortes por ano no mundo ${ }^{6}$. Estima-se que haja em torno de 1,4 bilhões de fumantes no mundo, somando cerca de seis trilhões de cigarros fumados por ano. No Brasil, aproximadamente $34 \%$ dos homens e $29 \%$ das mulheres são fumantes, consumindo cerca de 175 bilhões de cigarro por ano ${ }^{7}$. Um levantamento realizado no Brasil demonstra uma prevalência de $38 \%$ do consumo de cigarro na faixa de 18 a 24 anos $^{8}$.

Diversas pesquisas mostram que a população universitária corrobora com os 
dados supracitados. Uma revisão de literatura realizada por Wagner et al. (2008) ${ }^{9}$ referente aos estudos com a população universitária no período de 1997 a 2007 aponta que os jovens consomem álcool e tabaco com grande prevalência. Além disso, alguns estudos incluídos nesta revisão evidenciaram que o consumo se inicia antes do ingresso na universidade, e outros mostraram que há um aumento crescente durante o período universitário ${ }^{9}$. Os estudos nos fazem pensar que nem sempre o meio universitário é o ponto de partida para o consumo.

Entretanto, é preciso considerar que o meio acadêmico possibilita diversas mudanças na vida dos estudantes, como novas relações sociais e adoção de novos comportamentos. Isso vem frequentemente junto com situações próprias da adolescência, como alteração biológica e instabilidade psicossocial, tornando o universitário vulnerável a circunstâncias que colocam em risco sua saúde ${ }^{10}$. Por isso, a adoção ou a manutenção de hábitos saudáveis nessa fase pode representar um importante passo para a consolidação desses hábitos pelo resto da vida.

Desta forma, este estudo tem relevância ao tentar investigar e compreender as características e o perfil da população universitária da UFPel, preocupação que ainda não recebeu atenção de estudiosos. A partir da pesquisa, pretende-se chamar a atenção para futuras intervenções no combate ao tabagismo e ao álcool.

Consequentemente, o objetivo do presente estudo foi avaliar o consumo de álcool e tabagismo entre estudantes universitários de primeiro ano da Universidade Federal de Pelotas (UFPel).

\section{Metodologia}

Foi realizado um estudo transversal em uma amostra de estudantes que ingressaram na Universidade Federal de Pelotas (UFPel) no processo seletivo do verão de 2008. Para selecionar a amostra, realizamos um levantamento da relação de cursos, segundo o Departamento de Registros Acadêmicos (DRA-UFPEL), conforme as cinco áreas dos cursos de graduação, que são: Ciências Agrárias, Ciências Biológicas, Ciências Exatas, Ciências Humanas e os cursos do Instituto de Letras e Artes. Para obtermos uma amostra representativa e equivalente de cada área de estudo, sorteamos alguns cursos de maneira aleatória, totalizando dezesseis cursos.

O processo amostral foi realizado em múltiplos estágios; primeiramente a amostra foi estratificada segundo a grande área proporcional ao número de alunos ingressantes na área acadêmica. Em segundo lugar, foi realizado um sorteio aleatório dos cursos dentro de cada área, de forma a obter o número estimado de alunos, respeitando a proporcionalidade de cada área. Logo após foi realizada a seleção de todos os alunos ingressantes nos cursos sorteados. Por fim em relação ao tamanho amostral, foram investigados cerca de 500 estudantes, o que garantiu um nível de confiança de $95 \%$.

Inicialmente, foi realizado um estudo piloto no qual foram aplicados questionários em duas turmas da Escola Superior de Educação Física da UFPel que não haviam sido selecionadas para a amostra. A coleta de dados foi realizada por acadêmicos treinados no segundo semestre de 2008. Na sala de aula dos cursos selecionados, antes de o questionário ser entregue junto com o termo de consentimento livre e esclarecido, foram dadas instruções sobre o preenchimento do instrumento. Fizeram parte da amostra os alunos matriculados conforme o colegiado de curso e presentes em sala de aula no dia da entrevista. O instrumento utilizado no estudo foi um questionário autoaplicado com perguntas referentes aos hábitos de vida relacionados à saúde.

Para avaliação do consumo de álcool utilizou-se uma pergunta sobre a frequência em que o estudante bebia. Para estudantes que relataram algum tipo de consumo foi utilizado o questionário CAGE. Este instrumento é composto por quatro questões representadas pelas palavras-chave de cada letra: C - Cut Down (diminuir ingestão); A 
- Annoyed (irritado); G - Guilty (culpado); E - Eye-Opener (identificação de ressaca). No Brasil a validação deste questionário encontrou uma sensibilidade de $88 \%$ e uma especificidade de $83 \%^{11}$. Nas quatro perguntas temos opções de "afirmativa" ou "negativa". Quando se obteve duas ou mais afirmações, ocorre a confirmação da suspeita de uso abusivo de álcool. Além do questionário citado, perguntamos aos respondentes se o hábito de consumo de álcool foi iniciado antes ou depois do ingresso na universidade. Posteriormente, o consumo de álcool foi operacionalizado em dois grupos, sendo considerados "consumidores" aqueles indivíduos que relataram beber pelo menos uma vez ao mês e o outro grupo, "não consumidores", ou seja, aqueles indivíduos que nunca utilizam álcool.

Em relação ao tabagismo, utilizou-se questionário pré-testado ${ }^{12,13}$. Para aqueles que relataram "sim", foi questionado se os universitários adquiriram o vício antes ou depois do ingresso na universidade. $\mathrm{Na}$ análise, os alunos foram divididos em dois grupos: os fumantes, ou seja, aqueles que relataram consumir cigarro em festas ou fins-de-semana, e aqueles que fumam regularmente. Por outro lado, foram considerados não fumantes aqueles que não consomem cigarro, mesmo que já tenham sido fumantes.

Além das informações analisadas como desfecho nesse artigo também foram pesquisadas variáveis demográficas envolvendo sexo (masculino ou feminino), idade, tipo de escola no ensino médio (escola pública, escola particular, parcialmente em escola particular ou outras), local de moradia (com pais ou parentes, pensionato, república ou casa de estudantes, casa/ apartamento dividido com amigos, sozinho ou outros), nível socioeconômico (ABEP - Associação Brasileira de empresas de pesquisa - 2003 - www.abep.org) e aspectos comportamentais e de saúde, incluindo a autopercepção de saúde (excelente, muito boa, boa, regular ou ruim).

Os dados foram duplamente digitados no programa Epi-Info 6.0 e checados em relação a possíveis inconsistências. As análises estatísticas foram realizadas no pacote estatístico Stata 9.0. De início foram realizadas as análises descritivas (com médias e proporções) e, por fim, análises de associação através dos testes de qui-quadrado para heterogeneidade e Odds Ratio.

A pesquisa foi aprovada pelo Comitê de Ética em pesquisa da Escola Superior de Educação Física da Universidade Federal de Pelotas, recebendo aprovação sob protocolo no $022 / 2008$.

\section{Resultados}

Participaram do estudo 485 estudantes, sendo 53,9\% do sexo feminino. A média de idade dos alunos foi de 22,6 anos (DP = 7,3), sendo que $42,3 \%$ dos alunos tinham menos de 20 anos. A Tabela 1 descreve a amostra segundo características demográficas, socioeconômicas e de saúde. Cerca de 3/4 dos alunos estudaram em escola pública no ensino médio e aproximadamente $1 / 3$ não moram com os pais ou responsáveis. Em relação à autopercepção de saúde, 47,9\% dos entrevistados consideram sua saúde como "muito boa."

Em relação às áreas de conhecimento, a amostra ficou assim distribuída: Ciências Agrárias (3,5\%), Ciências Biológicas (27,8\%), Ciências Exatas e Tecnológicas (25,8\%), Ciências Humanas $(34,8 \%)$ e Letras e Artes $(8,1 \%)$. O curso de Direito apresentou maior número de alunos, representando $18,1 \%$ da amostra. O curso de Música foi aquele representado pelo menor número de alunos $(\mathrm{N}=6)$.

O consumo de álcool e o tabagismo estão descritos na Tabela 2 . O consumo de álcool, pelo menos uma vez ao mês, foi relatado por $75 \%$ da amostra, não mostrando diferença segundo o sexo.

Em relação ao tabagismo, 11,4\% dos estudantes homens relataram que fumam regularmente ou nos fins-de-semana. Este percentual foi menor no sexo feminino $(8,8 \%)$. Entre os estudantes entrevistados, 5,2\% disseram que já foram fumantes, mas pararam com o consumo, e 
Tabela 1 - Descrição da amostra segundo variáveis demográficas e socioeconômicas.

Table 1 - Description of the sample according to demographic and socioeconomic variables.

\begin{tabular}{|c|c|c|}
\hline Variáveis & $\mathrm{N}^{\mathrm{a}}$ & $\%$ \\
\hline \multicolumn{3}{|l|}{ Sexo } \\
\hline Masculino & 220 & 46,1 \\
\hline Feminino & 257 & 53,9 \\
\hline \multicolumn{3}{|l|}{ Idade (anos) } \\
\hline$<20$ & 204 & 42,3 \\
\hline 20 a 24 & 183 & 38,0 \\
\hline$>25$ & 95 & 19,7 \\
\hline \multicolumn{3}{|l|}{ Tipo de escola no ensino médio } \\
\hline Escola Pública & 358 & 74,7 \\
\hline Escola Particular & 112 & 23,4 \\
\hline Parcialmente em escola particular & 6 & 1,3 \\
\hline Outra & 3 & 0,6 \\
\hline \multicolumn{3}{|l|}{ Nível socioeconômico } \\
\hline A & 73 & 15,8 \\
\hline B & 227 & 49,2 \\
\hline$C / D$ & 161 & 34,9 \\
\hline \multicolumn{3}{|l|}{ Local de moradia } \\
\hline Pais ou parentes & 320 & 66,8 \\
\hline Pensionato, república & 20 & 4,2 \\
\hline Casa dividida com amigos & 62 & 12,9 \\
\hline Sozinho & 33 & 6,9 \\
\hline Outro & 44 & 9,1 \\
\hline \multicolumn{3}{|l|}{ Autopercepção de Saúde } \\
\hline Excelente & 89 & 18,5 \\
\hline Muito Boa & 230 & 47,9 \\
\hline Boa & 133 & 27,7 \\
\hline Regular & 26 & 5,4 \\
\hline Ruim & 2 & 0,4 \\
\hline Total & 485 & 100,0 \\
\hline
\end{tabular}

${ }^{\mathrm{a}} \mathrm{N}=485$

O número máximo de informações perdidas foi para a variável nível socioeconômico ( $\mathrm{N}=24)$.

The maximum number of missing values was for the variable socio-economic level $(N=24)$.

aproximadamente $65 \%$ da amostra relataram nunca ter fumado.

O risco para o alcoolismo foi investigado entre os alunos que relataram o consumo de álcool e 6,2\% destes apresentaram resultado positivo segundo o instrumento CAGE, sendo $7,7 \%$ entre os homens e $5,1 \%$ entre as mulheres (Figura 1).

Ainda entre aqueles acadêmicos que ingeriram álcool, a grande maioria $(92,7 \%)$ relatou este hábito como antecedendo ao ingresso na Universidade, não mostrando diferença importante segundo o sexo $(92,5 \%$ e $92,8 \%$ entre homens e mulheres, respectivamente). Tal quadro repetiu-se quando analisado o tabagismo. Apenas 2,5\% dos alunos do sexo masculino e $4,4 \%$ do sexo feminino adquiriram o hábito depois de ingressar na UFPel (Tabela 3).

A Tabela 4 mostra a associação entre o consumo de álcool e o tabagismo com variáveis demográficas, socioeconômicas e de saúde. O tabagismo apresentou uma relação direta com a idade, ou seja, quanto 
Tabela 2 - Consumo de álcool e tabaco entre estudantes da Universidade Federal de Pelotas (UFPel).

Table 2 - Consumption of alcohol and tobacco among students from the Federal University of Pelotas (UFPel).

\begin{tabular}{lcccccc}
\hline Variáveis & \multicolumn{2}{c}{ Total } & \multicolumn{2}{c}{ Masculino } & \multicolumn{2}{c}{ Feminino } \\
\cline { 2 - 7 } & $\mathrm{N}$ & $\%$ & $\mathrm{~N}$ & $\%$ & $\mathrm{~N}$ & $\%$ \\
\hline Consumo de álcool & & & & \multicolumn{2}{c}{$P=0,117$} & \\
$\quad$ Nunca bebeu & 118 & 24,6 & 55 & 25,5 & 63 & 24,7 \\
$\quad$ Mensal ou menos & 153 & 31,9 & 57 & 26,4 & 91 & 35,7 \\
$\quad$ 2-4 vezes por mês & 151 & 31,5 & 72 & 33,3 & 76 & 29,8 \\
$\quad$ 2-3 vezes por semana & 52 & 10,9 & 28 & 13,0 & 24 & 9,4 \\
$\quad$ 4 ou mais vezes por semana & 5 & 1,1 & 4 & 1,8 & 1 & 0,4 \\
\hline Total & 479 & 100,0 & 216 & 100 & 255 & 100 \\
\hline Consumo de tabaco & & & \multicolumn{4}{c}{$P=0,254$} \\
$\quad$ Nunca fumou & 307 & 65,5 & 139 & 65,6 & 163 & 65,5 \\
$\quad$ Experimentou, mas não regular & 89 & 19,0 & 38 & 17,9 & 51 & 20,5 \\
$\quad$ Fuma em festa ou final de semana & 23 & 4,9 & 8 & 3,8 & 14 & 5,6 \\
$\quad$ Já foi fumante, mas parou & 25 & 5,3 & 11 & 5,2 & 13 & 5,2 \\
$\quad$ Fumante atual & 25 & 5,3 & 16 & 7,6 & 8 & 3,2 \\
\hline Total & 469 & 100,0 & 212 & 100,0 & 249 & 100,0 \\
\hline
\end{tabular}

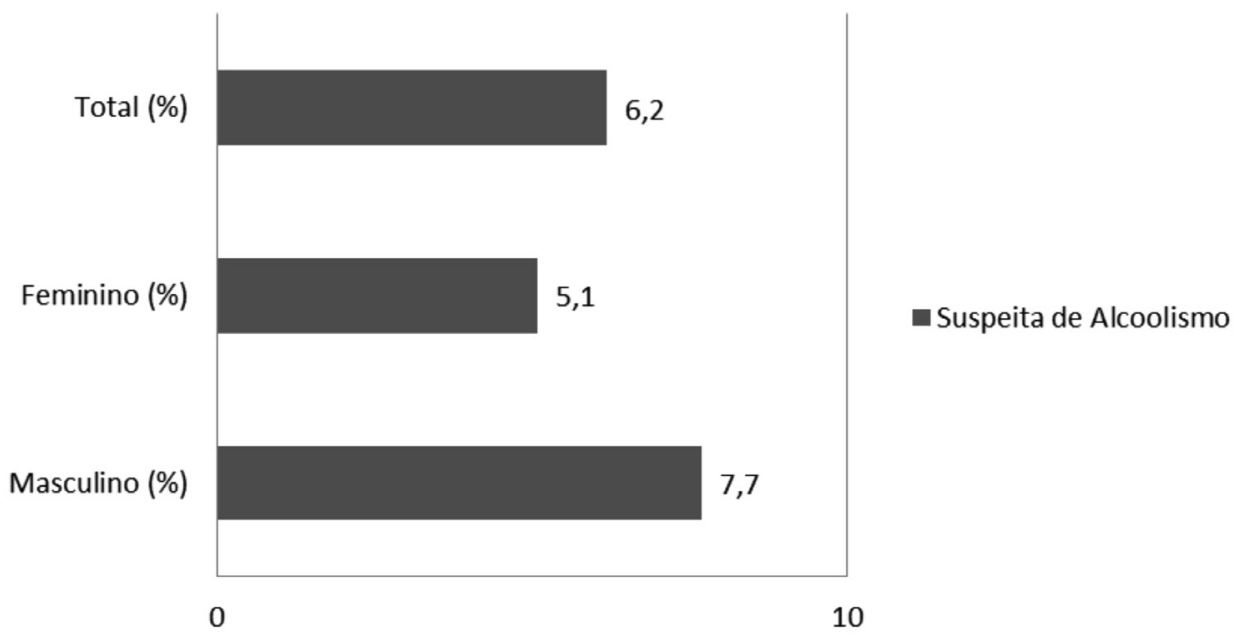

Figura 1 - Prevalência dos indicativos de problemas com álcool segundo o instrumento CAGE. C - Cut Down (diminuir ingestão); A - Annoyed (irritado); G - Guilty (culpado); E - Eye-Opener (identificação de ressaca).

Figure 1- Prevalence of indicatives of problems with alcohol according to the CAGE (C - Cut Down; A Annoyed; G-Guilty; E-Eye-Opener ) instrument.

mais velho o universitário maior foi a probabilidade de consumo e, em contraste, quanto menor a autopercepção de saúde maior foi a probabilidade do tabagismo. Os estudantes que moram sozinhos apresentaram maior prevalência de tabagismo $(24,2 \%)$, enquanto aqueles que dividem a moradia com amigos relataram maior consumo de álcool (93,3\%). Acadêmicos oriundos de escolas particulares no ensino médio apresentaram maior probabilidade de consumo de álcool e tabagismo. Da mesma forma, o grupo de nível socioeconômico A também apresentou maior probabilidade de consumir álcool e tabaco.

\section{Discussão}

O presente estudo é um dos primeiros a respeito do tabagismo e do consumo de 
Tabela 3 - Descrição da amostra segundo o momento que iniciou a consumir álcool e/ou cigarro.

Table 3 - Description of the sample according to alcohol consumption and smoking onset.

\begin{tabular}{|c|c|c|}
\hline Variáveis & Masculino N (\%) & Feminino $\mathrm{N}(\%)$ \\
\hline Quando Adquiriu o Hábito de Beber & \multicolumn{2}{|c|}{$\mathrm{P}=0,89$} \\
\hline Antes de ingressar na UFPel & $147(92,5)$ & $168(92,8)$ \\
\hline Depois de Ingressar na UFPel & $12(7,5)$ & $13(7,2)$ \\
\hline Total & $159(100,0)$ & $181(100,0)$ \\
\hline Quando Adquiriu o Hábito de Fumar & \multicolumn{2}{|c|}{$\mathrm{P}=0,62$} \\
\hline Antes de ingressar na UFPel & $39(97,5)$ & $42(95,6)$ \\
\hline Depois de Ingressar na UFPel & $1(2,5)$ & $2(4,4)$ \\
\hline Total & $40(100,0)$ & $45(100,0)$ \\
\hline
\end{tabular}

Tabela 4 - Associação do consumo de álcool e tabagismo com variáveis demográficas, socioeconômicas e de saúde. Table 4 - Association of alcohol consumption and smoking with demographic, socioeconomic, and health-related variables.

\begin{tabular}{|c|c|c|c|c|}
\hline \multirow[t]{2}{*}{ Variável } & \multicolumn{2}{|c|}{ Consumo de álcool $^{a}$} & \multicolumn{2}{|c|}{ Tabagismo $^{b}$} \\
\hline & $\%$ & OR (IC95\%) & $\%$ & OR (IC95\%) \\
\hline \multicolumn{5}{|l|}{ Sexo } \\
\hline Homens & 74,5 & 1,00 & 11,3 & 1,00 \\
\hline Mulheres & 75,3 & $1,22(0,77-1,96)$ & 8,8 & $0,82(0,42-1,62)$ \\
\hline \multicolumn{5}{|l|}{ Idade (anos) } \\
\hline$<20$ & 73,0 & 1,00 & 7,0 & 1,00 \\
\hline $20-24$ & 78,0 & $1,58(0,93-2,69)$ & 10,2 & $1,74(0,79-3,83)$ \\
\hline$\geq 25$ & 74,5 & $0,99(0,48-2,09)$ & 17,8 & $3,30(1,25-8,73)$ \\
\hline \multicolumn{5}{|c|}{ Tipo de escola no ensino médio } \\
\hline Pública & 73,0 & 1,00 & 9,3 & 1,00 \\
\hline Particular & 82,9 & $1,75(0,90-3,38)$ & 13,7 & $1,75(0,76-4,04)$ \\
\hline \multicolumn{5}{|l|}{ Local de moradia } \\
\hline Pais ou parentes & 70,7 & 1,00 & 8,1 & 1,00 \\
\hline Pensionato/república & 75,0 & $1,71(0,58-5,01)$ & 10,0 & $1,56(0,31-7,86)$ \\
\hline Dividida com amigos & 93,3 & $10,16(2,97-34,71)$ & 15,3 & $2,74(1,04-7,25)$ \\
\hline Sozinho & 84,4 & $2,20(0,78-6,23)$ & 24,2 & $3,40(1,19-9,74)$ \\
\hline Outro & 79,6 & $2,14(0,81-5,70)$ & 9,5 & $0,67(0,18-2,44)$ \\
\hline \multicolumn{5}{|l|}{ Auto percepção de saúde } \\
\hline Excelente & 77,3 & 1,00 & 8,2 & 1,00 \\
\hline Muito boa & 75,3 & $0,77(0,41-1,46)$ & 8,1 & $0,96(0,35-2,63)$ \\
\hline Boa & 72,0 & $0,74(0,37-1,48)$ & 12,3 & $1,87(0,68-5,20)$ \\
\hline Regular/ruim & 88,9 & $1,74(0,49-6,34)$ & 26,9 & $4,97(1,37-18,08)$ \\
\hline \multicolumn{5}{|l|}{ Nível socioeconômico } \\
\hline A & 81,9 & $1,99(0,85-4,69)$ & 12,6 & $2,00(0,61-6,60)$ \\
\hline B & 75,5 & $1,63(0,95-2,76)$ & 11,0 & $1,71(0,76-3,88)$ \\
\hline$C-D$ & 72,3 & 1,00 & 8,4 & 1,00 \\
\hline
\end{tabular}

${ }^{a}$ Consumo de álcool superior a 1 vez por mês (juntar as categorias 2-4 mês; 2-3 sem, etc)

${ }^{a}$ Alcohol consumption of more than once per month (categories 2-4 months, 2-3 weeks, etc.).

${ }^{\mathrm{b}}$ Tabagismo regular (fumante e fuma em festa).

${ }^{b}$ Regular smoking (Smoker and party smoker). 
álcool em uma amostra representativa de estudantes universitários no Brasil. Os resultados aqui apresentados podem servir de subsídios para futuras intervenções que objetivem estimular hábitos saudáveis entre os estudantes.

No Brasil, o tabagismo está diretamente relacionado a $30 \%$ dos casos de infarto agudo do miocárdio, $25 \%$ dos casos de acidente vascular cerebral, $85 \%$ das mortes por doença pulmonar obstrutiva crônica e pelo menos $90 \%$ das mortes por câncer de pulmão ${ }^{14}$. Assim como o tabaco, o consumo de álcool pode prejudicar a saúde, originar diversos problemas sociais e econômicos em nosso país. Por exemplo, um estudo que descreve trabalhos epidemiológicos sobre álcool no Brasil mostra que na análise de 130 processos de homicídios ocorridos em Curitiba, mais de $50,0 \%$ das vítimas e $58,9 \%$ dos autores dos crimes estavam sob efeito de álcool. O mesmo artigo relata que $27,2 \%$ das vítimas de acidentes de trânsito, em quatro capitais, apresentaram alcoolemia superior ao limite permitido na época do estudo ${ }^{4}$.

Algumas limitações em nosso estudo precisam ser discutidas. Primeiramente, alguns alunos não foram encontrados em sala de aula. Tal desconexão ocorreu porque a amostra foi calculada de acordo com os números de vagas de cada curso e não pelos alunos matriculados regularmente no semestre em que a pesquisa foi realizada. Em segundo lugar, por mais que os estudantes fossem esclarecidos sobre a importância da investigação, em alguns casos a falta de comprometimento dos mesmos com a pesquisa representou perdas em determinadas questões, considerando o fato de o instrumento ser autoaplicado.

A amostra do nosso estudo apontou que cerca de $2 / 3$ pertenciam aos níveis socioeconômicos A e B. Um estudo realizado no curso de medicina do Centro de Ciências da Saúde (CCS) da UFES mostra que, segundo a metodologia da ABIPEME, por volta de $90 \%$ dos estudantes pertenciam às classes A e $\mathrm{B}^{15}$. Outrossim, um estudo de base populacional realizado na cidade de Pelotas/ RS, demonstra que em torno de $68,0 \%$ da amostra pertenciam às classes $\mathrm{C}$ e $\mathrm{D}^{16}$. Portanto, assim como em outros estudos, nossos resultados confirmam a tendência de predominância de alunos de classes mais favorecidas na ocupação de vagas na UFPel.

Um estudo realizado em Pernambuco comparou universitários ingressantes e do último semestre quanto ao hábito de fumar. $\mathrm{O}$ estudo pernambucano mostrou que o uso frequente de cigarro obteve prevalência de $7,1 \%$ e $11,0 \%$ no primeiro e último semestres, respectivamente. O consumo de álcool (referente ao ultimo mês) foi de 52,3\% e $67,5 \%$ no primeiro e último semestres, respectivamente ${ }^{17}$. Os dados apresentados entre os alunos ingressantes da UFPel são comparáveis aos alunos formandos do estudo acima citado. Sendo assim, ambos os estudos salientam a necessidade de intervenções para que essas prevalências não se tornem mais expressivas no decorrer dos anos. Em nossa amostra, por não termos entrevistado alunos formandos, a comparação entre os diferentes momentos da vida universitária não pôde ser realizada.

Um estudo transversal realizado na faculdade de medicina da UFPel em 2002, com amostra de 447 alunos, apresentou tendência positiva entre o tabagismo e o ano do curso, sendo que no primeiro ano apenas 5,0\% consumiam tabaco, e no último ano $15,0 \%{ }^{15}$. A descrição demográfica do presente estudo assemelha-se ao citado anteriormente, porém na nossa pesquisa os resultados de tabagismo foram alarmantes, pois há uma comparação entre os alunos do primeiro semestre com os do quarto ano do curso de medicina (UFPel). Com isso, apesar de as prevalências estarem diminuindo no decorrer dos anos, ainda se torna imprescindível o planejamento de mais medidas antitabágicas para se evitar que o jovem inicie o hábito de fumar no âmbito escolar e universitário ${ }^{5}$.

Um estudo realizado na Universidade Federal do Amazonas entre acadêmicos dos cursos de farmácia, medicina e odontologia mostrou um consumo de álcool na vida de $87,7 \%$, com prevalência discretamente superior entre indivíduos do nível socioeconômico $\mathrm{B}^{18}$. No presente estudo, os estudantes 
que pertenciam ao nível socioeconômico A e B tinham maior probabilidade de consumir álcool e tabaco quando comparados aos níveis $\mathrm{C}$ e $\mathrm{D}$.

Daqueles que consomem álcool, segundo o questionário Cage, 6,2\% apresentaram uma suspeita de alcoolismo. Um estudo realizado com 3.644 universitários de ambos os sexos do município de Jequié, BA, apresentou um resultado mais expressivo, com $13,4 \%$ de suspeita de alcoolismo ${ }^{11}$. Já em uma pesquisa realizada em 16 escolas de Porto Alegre, em turmas de terceiro ano, o Cage foi positivo para $8,3 \%$ da amostra. A análise em turmas de terceiro ano não mostrou diferença significativa em relação a escola pública ou privada ${ }^{19}$. Estudo de base populacional na cidade de Pelotas/RS apresentou uma prevalência de 5,6\% de alcoolismo, segundo o instrumento CAGE $^{16}$. Em Rio Grande, RS, entre os que bebem, 2,5\% eram dependentes de álcool, segundo CAGE ${ }^{20}$.

A grande maioria dos estudantes relatou o primeiro contato com o cigarro e a bebida antes do ingresso na universidade. Ao analisarmos a nossa amostra, percebemos que $42,3 \%$ possuem menos de 20 anos. Uma pesquisa com universitários em Gurupi (TO) mostrou que o primeiro contato com o cigarro ocorre por volta dos 17 anos $^{21}$. Corroborando este achado, uma pesquisa realizada na faculdade de medicina de Passo Fundo verificou que $69,2 \%$ dos fumantes iniciaram o tabagismo entre 15 e 19 anos $^{22}$. Outro estudo realizado em Guayaquil identificou o uso de álcool e outras drogas, antes do ingresso na universidade, ocorrendo entre os 10 e 18 anos $^{3}$.

Conseguir uma diminuição nas prevalências de tabagismo e consumo de álcool da UFPel poderá acarretar em impactos na saúde pública. Patkar et al. (2003) ${ }^{23}$ discutiram a importância da diminuição das prevalências de tabagismo em estudantes de medicina e enfermagem, afirmando que esta diminuição pode causar um impacto na saúde pública, pois a adoção de hábitos de vida saudáveis pelos estudantes e futuros profissionais,podem auxiliar no manejo de pacientes quando iniciarem a carreira profissional.

O tabagismo está associado com a idade dos universitários. Um estudo realizado em São Paulo apresentou resultados semelhantes $^{24}$. A pesquisa realizada na faculdade de medicina (UFPel), apesar de não se mostrar associada com a idade, descreve que existe uma consistência na literatura a respeito da tendência linear direta entre tabagismo e o ano do curso ${ }^{5}$. Desta forma, se não houver algum tipo de intervenção, o aumento do tabagismo pode ser inevitável no decorrer dos anos. Outro fator importante em relação à idade e o tabagismo é que existem estudos comprovando que o indivíduo que fuma dos 12 aos 18 anos torna-se dependente da nicotina até os 19 anos, época em que está construindo sua personalidade ${ }^{10}$.

A associação do tabagismo e do consumo de álcool com o local de moradia pode ser explicado pelo fato de estes estudantes estarem vivenciando pela primeira vez a experiência de viver longe dos pais, com isso ocasionando uma possível ruptura de alguns hábitos e a adoção de novos estilos de vida ${ }^{10}$

Indivíduos que perceberam sua saúde como Regular ou Ruim apresentaram maior ocorrência de tabagismo. Considerando o fato de o cigarro possuir mais de 4000 substâncias químicas que podem ser identificadas na fumaça e que são nocivas ${ }^{7}$, aliado à forte mídia contra o tabagismo, parece clara a "autocondenação" do fumante sobre o seu hábito e a consciência do prejuízo para a sua saúde.

\section{Conclusão}

O tabagismo e o consumo de álcool apresentaram prevalências elevadas entre alunos ingressantes na UFPel. Os dados sugerem a necessidade de intervenções no meio acadêmico. Entretanto, cabe destacar que ações para a população adolescente como um todo devem ser priorizadas, pois mais de $90,0 \%$ dos alunos adquiriram os hábitos antes de ingressar na universidade. 


\section{Referências}

1. Prata PR. A transição epidemiológica no Brasil. Cad Saúde Pública 1992; 8: 168-75.

2. Noto AR, Galduróz JCF. Use and Prevention of Psychotropic Drugs in Brazil. Ciência \& Saúde coletiva 1999; 4(1): 145-51.

3. Chavez KAP, O'Brien B, Pillon SC. Drugs Use and Risk Behavior In a University Community. Rev Latino-am Enfermagem 2005; 13(número especial): 1194-200.

4. Galduróz JCF, Caetano R. Epidemiologia do uso de álcool no Brasil. Rev Bras Psiquiatr 2004; 26(S1): 3-6.

5. Menezes AMB, Hallal PC, Silva F, Souza M, Paiva L, D'Ávila A et al. Tabagismo em estudantes de medicina: tendências temporais e fatores associados. J Bras Pneumol 2004; 30(3): 223-8.

6. Fraga S, Ramos E, Barros H. Uso de tabaco por estudantes adolescentes portugueses e fatores associados. Rev Saúde Pública 2006; 40(4): 620-6.

7. Duncan BB, Schmidt MI, Giugliani ERJ. Medicina Ambulatorial: condutas de atenção primária. Achutti AC, Rosito MHE, Achutti VAR (eds). Porto Alegre: Artmed; 2004.

8. Neto ASM, Cruz ÁA. Smoking among school adolescents in Salvador (BA). J Pneumol 2003; 29(5): 264-72.

9. Wagner GA, Andrade AGD. Uso de álcool, tabaco e outras drogas entre estudantes universitários brasileiros. Rev Psiq Clin 2008; 35: 48-54.

10. Vieira VCR, Priore SE, Ribeiro SMR, Franceschini SCC, Almeida LP. Perfil socioeconômico, nutricional e de saúde de adolescentes recém-ingressos em uma universidade pública brasileira. Rev Nutr 2002; 15: 27382.

11. Rios PAA, Matos AM, Fernandes MH, Barbosa AR. Alcoholic Ingestion and Alcohol Abuse in University Students at Jequié-BA. Rev Saúde Col 2008; 4(2): 105-16.

12. Victora CG, Barros FC. Cohort Profile: The 1982 Pelotas (Brazil) Birth Cohort Study. Int J Epidemiol 2006 2006; 35(2): 237-42.

13. Victora CG, Hallal PC, AraÃojo CLP, Menezes AMB, Wells JCK, Barros FC. Cohort Profile: The 1993 Pelotas (Brazil) Birth Cohort Study. Int J Epidemiol 2008 1, 2008; 37(4): 704-9.

14. Silva MAM, Rivera IR, Carvalho ACC, Guerra Júnior AdH, Moreira TCA. Prevalência e variáveis associadas ao hábito de fumar em crianças e adolescentes. J Pediatr 2006; 82: 365-70.
15. Pereira DS, Souza RS, Buaiz V, Siqueira MM. Uso de substâncias psicoativas entre universitários de medicina da Universidade Federal do Espírito Santo. J Bras Psiq 2008; 57: 188-95.

16. Noal RB, Menezes AMB, Canani SF, Siqueira FV. Ronco habitual e apnéia obstrutiva observada em adultos: estudo de base populacional, Pelotas, RS. Rev Saúde Pública 2008; 42: 224-33.

17. Franca C, Colares V. Estudo comparativo de condutas de saúde entre universitários no início e no final do curso. Rev Saúde Pública 2008; 42(3): 420-7.

18. Lucas ACS, Parente RCP, Picanço NS, Conceição DA, Costa KRC, Magalhães IRS, et al. Uso de psicotrópicos entre universitários da área da saúde da Universidade Federal do Amazonas, Brasil. Cad Saúde Pública 2006; 22: 663-71.

19. Trois CC, Frantz BC, Yaluk JB, Taroncher CA, Schneider W, Schonell LHB et al. Prevalência de CAGE positivo entre estudantes de segundo grau de Porto Alegre, Rio Grande do Sul, Brasil, 1994. Cad Saúde Pública 1997; 13: 489-95.

20. Primo NLNP, Stein AT. Prevalência do abuso e da dependência de álcool em Rio Grande (RS): um estudo transversal de base populacional. Rev Psiq Rio Grande do Sul 2004; 26: 280-6.

21. Rodrigues ESR, Cheik NC, Mayer AF. Nível de atividade física e tabagismo em universitários. Rev Saúde Pública 2008; 42: 672-8.

22. Stramari LM, Kurtz M, Silva LCC. Prevalência e fatores associados ao tabagismo em estudantes de medicina de uma universidade em Passo Fundo (RS). J Bras Pneumol 2009; 35: 442-8.

23. Patkar AA, Hill K, Batra V, Vergare MJ, Leone FT. A Comparison of Smoking Habits Among Medical and Nursing Students. CHEST 2003; 124(4): 1415.

24. Silva LVER, Malbergier A, StempliukVA, Andrade AG. Factors Associated with Drug and Alcohol Use Among University Students. Rev Saúde Pública 2006; 40(2): 2808.

Recebido em: 12/04/2010

Versão final apresentada em: 13/04/2011

Aprovado em: 23/07/2011 